\title{
COMETS, METEORITES AND INTERPLANETARY DUST
}

\author{
D. E. Brownlee \\ University of Washington \\ Dept. of Astronomy \\ Seattle, Washington 98195 \\ U.S.A.
}

\begin{abstract}
Cometary debris of all sizes impacts the Earth but it is likely that only particles the size of dust survive atmospheric entry and are collected as meteoritic samples. Conventional meteorites and a substantial fraction of collected interplanetary dust particles appear to be asteroidal debris. Nearly half of the collected interplanetary particles have properties consistent with cometary material and resemble Halley dust that has lost the majority of its carbon and nitrogen. These particles might be aggregates of presolar grains and they provide some insight into the properties of interstellar grains.
\end{abstract}

\section{INTRODUCTION}

New information from the 1986 Halley flybys, meteorites, micrometeorites, and IRAS observations of interplanetary dust, have provided new insights into the relationship between meteoritic samples, comets and asteroids. The measured and inferred properties of cometary particles also provide new clues on the possibility that comets contain preserved presolar interstellar grains.

\section{METEORITES}

Conventional meteorites have been the traditional source of extraterrestrial material investigated in the laboratory. Meteorites, for the most part are primitive solar system materials and their chemical, mineralogical and isotopic properties provide a wealth of information on early solar system processes, materials and environments (Kerridge 1988). Conventional meteorites are large, critically important samples but they have a major limitation. They appear to be samples of only a small number of the solar system's comet and asteroid population. It likely that important classes of minor planets are not represented at all in meteorite collections.

Conventional meteorites are not a representative sample of the solar system's minor planet inventory because of selection effects associated with orbital transfer to Earthcrossing orbits and survival of hypervelocity entry into the atmosphere. Due to their size, meteorites can only be perturbed to Earth-crossing orbits by purely gravitational interactions. This is not a serious selection effect for cometary objects that are commonly released on Earth-crossing orbits, but it is important for asteroidal fragments. Meteorites from the asteroid belt reach the Earth after a series of gravitational perturbations. In the majority of cases the perturbations may initiate in chaotic zones associated with orbital resonances with Jupiter (Wisdom 1983). Wetherill (1985) has made a strong arguments that the the most common chondrite groups originate from the 3:1 Kirkwood gap, a very small region of the asteroid belt. The most serious selection effect on conventional meteorites is due to the stresses of atmospheric entry. To survive entry as an intact centimeter object, a meteoroid must strong. The peak atmospheric ram pressure experienced by centimeter objects results in disintegration of fragile materials and partial fragmentation of even the strongest stony meteorites. The most friable recovered meteorites are still rather strong rocks and are much stronger than cometary materials observed as typical meteors. Typical 
cometary meteoroids in streams fragment when the dynamic ram pressure exceeds $10^{5}$ dynes $\mathrm{cm}^{-2}$ (Verniani 1960) and yet the weakest chondrites have crushing strengths orders of magnitude higher. The most fragile stream meteors are the Draconids with strengths near $10^{3}$ dynes $\mathrm{cm}^{-2}$ (Sekanina 1985). From atmospheric entry considerations it is clear that typical cometary meteoroids cannot become conventional meteorites. If comets do not contain strong components then no cometary samples would survive entry as particles larger than centimeter size.

An asteroidal origin for conventional meteorites is supported by mineralogical evidence that even the most chemically primitive meteorite classes have experienced at least mild metamorphism. The most volatile rich chondrites, those that most closely match solar abundances, show evidence for aqueous alteration. The type 1 (CI) chondrites even have conspicuous sulfate veins deposited from aqueous solution (Kerridge and Bunch 1979). Evidence for warm episodes in the history of all meteorites is strong evidence against origin from comets, bodies that sublime when heated above cryogenic temperatures. An additional argument that meteorites are derived from inner solar solar system bodies is that the abundance of implanted solar wind in brecciated chondrites is consistent with exposure conditions within $5 \mathrm{AU}$ (Anders 1975).

The evidence suggests that most and probably all meteorites are samples of asteroids but comparison with asteroid reflectance data shows that meteorites are not representative sample of the main belt asteroids. Over $70 \%$ of meteorites falls are ordinary chondrites (Wasson 1974) and yet spectral reflectance studies show that such materials are rare in the main belt asteroids (Gaffey and McCord 1979). The majority of asteroids have rather flat spectral reflectance curves similar to the carbonaceous chondrites. The carbon rich carbonaceous chondrites constitute only $2.6 \%$ of meteorite falls. In this regard it is highly significant that roughly $85 \%$ of the interplanetary dust particles that are collected and analyzed are more similar to the carbonaceous chondrites than any other meteorite group.

It is likely that there are no cometary materials that have become conventional meteorites. It also appears that the typical materials in the asteroid belt also do not commonly produce meteorites and are highly under represented in meteorite collections.

\section{INTERPLANETARY DUST}

\subsection{Samples}

Interplanetary dust (IDP) is particulate material that must include samples of the parentbodies that produce meteorites but they also appear to include samples of materials not contained in meteorite collections. Collected samples range in size from a few microns to a millimeter in diameter. The best preserved samples are the $10 \mu \mathrm{m}$ size particles collected from the stratosphere with U2 aircraft. Larger particles are collected from polar ice and deep sea sediments (Brownlee 1985). Most of the larger particles ( $>100 \mu m$ ) melt during atmospheric entry but some properties such as elemental and isotopic composition are preserved.

\subsection{Selection effects}

Collected dust particles are expected to be a more representative sampling of the solar systems minor planet population because of less severe selection effects during orbital evolution and atmospheric entry. Orbits of dust particles decay due to Poynting-Roberston drag and all dust particles generated beyond $1 A U$ can become Earth-crossers if they are not destroyed by mutual collisions. For this reason it is expected that all classes of solar system minor bodies should generate dust that eventually reaches the Earth. Once at the Earth there are of course atmospheric selection effects but the survival criteria are different than those applicable to centimeter and larger objects. A critical difference for survival of cometary matter is that small particles are not subjected to dynamic ram pressures as high as the crushing strength of cometary meteors. Particles of $10 \mu \mathrm{m}$ size decelerate from cosmic velocity above $90 \mathrm{~km}$ where the ambient air density is sufficiently low that the maximum ram pressure does not reach $10^{5}$ dynes $/ \mathrm{cm}^{2}$, the typical strength of cometary meteors. Even Draconid particles should survive without fragmentation for sizes of $10 \mu \mathrm{m}$ and smaller. Larger and denser particles retain hypervelocity speeds into denser layers of 
the atmosphere and they are accordingly exposed to higher ram pressures.

\subsection{Origin}

Interplanetary dust particles are purged from the solar system by Poynting-Robertson drag and collisions. If the meteoroid complex is maintained in equilibrium against losses, new particles must be injected into the interplanetary medium on time scales of less than 100,000 years. The major sources of dust are asteroids and comets. Comets have traditionally been believed to be the dominant source of new particles because comets are the major source of meteors and until the IRAS observations, there was no observational evidence for the presence of dust in the asteroid belt. Due to light pressure effects, most of the dust released from long period comets travels on hyperbolic orbits and is lost from the solar system (Harwit 1963). Short period comets do release dust into bound orbits but the amount of observed dust from these objects appears to be insufficient to counter the $10^{7} \mathrm{gs}^{-1}$ loss rate due to collisions and the Poynting-Robertson effect (Kresak 1987). It is possible that much of the comet dust in the solar system was produced by extinct comets or that the major comet dust source is debris from progressive communition of larger cometary meteoroids (Kresak 1987) not directly observable in cometary comae.

For full interpretation of the cometary particles that are collected as micrometeorites, it is important to know whether the majority of particles are samples of only one or two comets or a large number of comets. Kresak's study (1987) indicates that over the past 200 years, particles from $\mathrm{P} /$ Encke have dominated the input to the zodiacal cloud and that over $90 \%$ of the total input has come from this comet as well as $\mathrm{P} / \mathrm{Halley}, \mathrm{P} / \mathrm{Brorsen}$, and $\mathrm{P} /$ Biela. Since the present dust population is the integral result of emissions over $10^{4}$ to $10^{5}$ years, prediction of the dominant sources of interplanetary comet dust is quite uncertain.

Some fraction of IDPs must be debris from the asteroids but the ratio of cometary to asteroidal interplanetary particles at $1 \mathrm{AU}$ is unknown. The discovery of IR emission from asteroid dust and the IRAS dust bands (Low et al. 1983) has lead to a renewed interest in asteroids as a source of interplanetary particles. Asteroids appear to be an important source of interplanetary particles that reach the Earth and it is possible that they are the major source (see Dermott and Nicholson in this symposium). The asteroid particles that spiral into $1 \mathrm{AU}$ should be relatively representative samples of the surface area of the asteroid belt but there may be a bias towards debris from asteroid families such as those that apparently produce the IRAS dust bands (Sykes and Greenberg 1986).

\subsection{Types}

The properties of micrometeorites collected in the stratosphere have been described elsewhere (Bradley et al. 1988, Mackinnon and Reitmeijer 1987, Sandford 1987 and Brownlee 1985). The particles can be classified into two broad groups that are primarily distinguished by mineralogical and morphological differences. One of the groups is dominated by hydrated minerals and the other is dominated by anhydrous minerals. Both particle types are black fine grained materials that generally match the elemental composition of carbonaceous chondrites within a factor of two for major and minor elements.

3.5.1. The Hydrous Group. This group, also called CS (chondritic smooth), is probably of asteroidal origin or at least it appears to be dominated by asteroidal particles. The CS particles are usually nonporous and have smooth, cracked, plately or fiberous surface textures. The dominant minerals are hydrated silicates and there are two subgroups one of which is dominated by serpentine and the other by smectite-like phases. Many of these particles contain evidence for aqueous alteration of the type that has altered the CI and CM (C1 and C2) carbonaceous chondrites. This evidence includes depletion of Ca (Schramm et al. 1988), presumably to water soluble phases, and the presence of carbonates and clusters of magnetite grains. The magnetite clusters are identical to those in CI chondrites formed by aqueous alteration. The evidence for aqueous alteration provides a strong argument against a cometary origin for particles in the CS class. It is possible that the progenitors of the CS particles were porous and fragile and that aqueous alteration produced the hydrated minerals, closed voids and strengthened the particles. Many of the CS particles are nearly identical to CI and CM chondrites and may be samples of the parentbodies of these meteorites. 
3.5.2. The Anhydrous Group. This group (also called CP or chon dritic porous) is dominated by anhydrous minerals and is probably of cometary origin (Bradley and Brownlee 1986). Unlike all conventional meteorites many of the CP particles are porous with porosities that sometimes exceed $50 \%$. The typical micro-porosity of chondrites is only a few percent. The open microstructures of $C P$ particles is inconsistent with a history of compaction unless sublimable phases once filled void spaces. The porous aggregate structure of the CP particles is unique among meteoritic samples but it is consistent with the friable properties attributed to cometary meteors. The dominant constituents of CP particles are olivine, pyroxene, iron sulfide, glass and carbon. These materials occur as submicron equidimensional grains. Some of the submicron grains are solid microaggregates ("tarballs") of mineral grains less than $0.1 \mu \mathrm{m}$ in diameter. Carbon occurs as discrete submicron grains and as coatings on grains. Nearly of the carbonaceous matter is amorphous and generally resembles the aromatic rich kerogen found in carbonaceous chondrites. Graphite is exceedingly rare. Raman studies indicate the presence of carbonaceous layers on silicates that obscure the the characteristic Raman silicate bands (Wopenka 1988). Direct observation by electron microscopy of sectioned particles indicates however that such coatings are thin in the range of $100 \mathrm{~A}$.

\section{COMET DUST}

The 1986 Halley flybys provided the first direct compositional measurements of comet dust. The PIA and PUMA mass spectrometers flown on Giotto and the VEGA spacecraft provided thousands of time-of-flight mass spectra of individual micron and submicron particles (Kissel 1986a,b). The integral of these spectra implies a bulk elemental composition of Halley solids that is consistent with chondrites except that the carbon and nitrogen abundances are greatly enhanced. The abundances of these two elements relative to silicon appears to be approximately solar (Jessberger 1988). Roughly a third of the particles are dominated by $\mathrm{H}, \mathrm{C}, \mathrm{N}$ and $\mathrm{O}$ and are called "CHON". The most common particles are a mix of the light elements and the major chondritic "silicate" elements $\mathrm{Mg}$, Si and $\mathrm{Fe}$. The remainder of the particles contain only low carbon abundances and typically have compositions consistent with mixtures of silicates and sulfur. An important property of the silicate composition particles is that many of them are Fe free magnesium silicates. Presumably these are composed of forsterite or enstatite, minerals that condense at high temperatures from solar composition gas. The range in composition of micron and submicron Halley particles provides a powerful means of comparing Halley with other primitive solar system materials. The micron scale dispersion of $\mathrm{Mg}$ and $\mathrm{Fe}$ in Halley is clearly distinct from that observed in carbonaceous chondrites meteorites but it is a fairly good match with the anhydrous class of interplanetary dust (Jessberger et al. 1988; Lawler et al. 1988). Like the interplanetary particles, Halley dust shows a wide range of $\mathrm{Mg} / \mathrm{Fe}$ ratios compared to those in the fine grained fraction of CI and CM meteorites.

With the exception of $C$ and $N$, the Halley particles have bulk compositions similar to primitive chondrites and interplanetary dust but the fine scale heterogeneity indicates that Halley is mineralogically distinct from the carbon rich chondrites and many of the hydrated types of IDPs. The anhydrous dust particles are the best match to the Halley data and their mineralogical composition is also consistent with fine structure seen in $10 \mu \mathrm{m}$ silicate emission observed in Halley (Bregman 1987). If indeed Halley is composed of anhydrous minerals this would imply that Halley is a mix of low temperature ice and volatiles mixed at fine scale with rather high temperature minerals such as pure Mg olivine. Condensation of this phase from the solar nebula occurs at temperatures above $1200 \mathrm{~K}$. The glaring discrepancy between the Halley measurements and collected samples is the abundance of carbon. The $\mathrm{C} / \mathrm{Si}$ atomic ratio in Halley dust is estimated by Jessberger et al. to be 4.4 , a value that is six times higher than CI chondrites and at least two and a half the mean value estimated for interplanetary dust. These differences could fundamental or is possible that much of the carbonaceous matter is Halley dust is relatively volatile and is lost in collected dust samples either during residence in the interplanetary medium or during atmospheric entry. 


\section{INTERSTELLAR DUST}

Anomalous isotopic compositions of minor components isolated by chemical, mechanical and thermal means, indicate that meteorites contain at least trace quantities of presolar matter that has retained isotopic memories of presolar processes. The recent discovery of small isotopically anomalous $\mathrm{SiC}$ grains in the Murray meteorite is evidence that minute qualities of genuine presolar interstellar grains are preserved (Zinner et al. 1987). The presence of deuterium rich nuggets in IDPs is also evidence for presolar grains (McKeegan et al. 1985). If asteroidal meteorites contain interstellar dust is is likely that cometary matter should contain higher abundances of presolar solids and might even be dominated by such materials. Greenberg (1986) has proposed such a model and details what cometary matter composed of interstellar grains would be like. With the Halley results and the IDP data it is now possible to make rather detailed comparisons with the Greenberg model. A major prediction of the model was high carbon abundance in cometary grains. This is consistent with the in-situ Halley measurements. The laboratory dust studies do not reveal such high average carbon abundance but this is easily reconciled if part of the carbonaceous is moderately volatile. The model also predicts that carbon should occur as thick radiation processed mantles covering prolate silicate cores. Some of the Halley particles could have such a structure but there is no direct evidence that this is the case. Nearly a third of the particles are nearly pure silicate and could not have thick mantles. A significant fraction of the submicron particles are nearly pure CHON material and do not appear to have substantial silicate cores. The hundreds of collected interplanetary particles do not have the precise morphology predicted by Greenberg but it is possible that the differences could be minimized by either processing the comet or particles or by mild metamorphism of the model. The collected cp particles are aggregates of rather equidimensional submicron grains and prolate grains are relatively rare. The need for prolate interstellar grains is to provide alignment and polarization. Instead of solid rod-like grains proposed in the model, it is entirely possible that prolate interstellar grains could be end-to-end clusters of two or more equidimensional grains such as those found in interplanetary dust. The Raman measurements indicate that silicate grains in IDPs are covered with carbonaceous material but imaging of the grains shows that such films are typically less than $200 \mathrm{~A}$, thinner than predicted by the model. This difference could be due to evolutionary processes. An intriguing possibility is that some of IDP grains called "tar balls" could have properties similar to core-mantle grains. These grains do not consist of a single silicate core surrounded by a thick mantle but rather they contain hundreds of $100 \mathrm{~A}$ size cores embedded in a carbonaceous mass.

\section{FUTURE WORK}

Results from the Halley flybys both corroborate the belief that comets are primitive materials and strengthen the notion that comets may be composed of presolar grains. Significant progress on the comet-interstellar grain connection can be made if proven samples of cometary material are subjected to laboratory analysis. The best samples would be collected directly from a comet nucleus such as is proposed by the Rosetta mission planned by the European Space Agency. The second best samples are interplanetary dust particles but it is imperative that means be developed for positive distinction of cometary and asteroidal samples. Information for such distinction should come from proposed missions such as the CRAF comet rendezvous proposed by NASA and the Cosmic Dust Collection Facility proposed for the NASA spacestation. At present it is important to persue the laboratory studies of probable cometary particles and to carefully evaluate data and models of cometary and interstellar grains. One of the obvious aspects to investigate is the implication that silicates in comets are anhydrous. Information on this problem can obtained for cometary and interstellar dust by careful study of the $10 \mu \mathrm{m}$ "silicate" feature.

To provide new insight into early solar system processes it is also pertinent carfully investigate the differences between asteroid and comet samples. The most primitive asteroid samples contain hydrated minerals while it is possible that cometary silicates are anhydrous. This could be an indication that low temperature equilibration with solar nebula gas only occured in the inner solar system. It is also possible however, that hydrated phases in 
asteroids formed entirely by parent body processes such as chemical weathering of silicates in contact with ice or water. The ability to compare detailed isotopic, chemical and mineraolgical differences between asteroid and comet samples should provide fundamental insights into properties of the solar nebula over a wide range of solar distance.

\section{REFERENCES}

Anders, E. 1975, Icarus, 24, 363.

Bradley, J. P. and Brownlee, D. E. 1986, Science, 231, 1452.

Bradley, J. P., et al. 1988, in Meteorites and the Early Solar System, eds. J. Kerridge and M. Mathews, Univ. of Arizona press.

Bregman, J. D. 1987, Astron. Astrophys., 187, 616.

Brownlee, D. E. 1985, Ann. Rev. Earth Planet. Sci., 13, 147.

Sykes, M. V., and Greenberg R. 1986, Icarus, 65, 51 .

Gaffey, M. J. and McCord, T. B. 1979, in Asteroids, ed. T. Gehrels, U. Arizona press, pp 688-723.

Greenberg, J. M. 1982, in Comets, ed. L. L. Wilkening, U. Arizona Press.

Grun, E., et al. 1986, lcarus, 62, 244.

Harwit, M. 1963, J. Geophys. Res., 68, 2171.

Jessberger, E. K. et al. 1988, Nature, 321, 691.

Kissel, J., et al. 1986a, Nature, 321, 280.

Kissel, J., et al. $1986 \mathrm{~b}$, Nature, 321, 336.

Kerridge, J. F. 1988, Meteorites and the The Early Solar System, Univ. of Arizona Press.

Kerridge, J. F. and Bunch, T. E. 1979, in Asteroids, Ed. T. Gehrels, U. Arizona Press, pp. $745-764$.

Kresak, L. 1987, Pub. Astron. Inst. Czechoslovak Acad. Sci., 67, 265.

Lawler, M. 1988, Icarus, in press.

Low, F. J. 1984, Astrophys. J. Lett., 278, 19.

Mackinnon, I. D. R. and Reitmeijer, F. J. M. 1987, Rev. Geophys., 25, 1527.

McKeegan, K. D. et al. 1985, Geochim. Cosmochim. Acta, 49, 1971.

Sandford, S. A. 1987, Fund. Cosmic Phys., 12, 1.

Schramm, L. S., et al. 1988, Meteoritics, in press.

Sekanina, Z. 1985, Astron. J., 90, 827.

Sykes, M. V. and Greenberg, R. 1986, Icarus, 65, 51.

Verniani, F. 1960, Space Sci. Rev., 10, 230.

Wasson, J. T. 1974, Meteorites (316 pages) New York: Springer Verlag.

Wetherill, G. W. 1985, Meteoritics, 20, 1.

Wopenka, B. 1988, Earth and Planet. 'Sci. Lett., 88, 221.

Wisdom, J. 1983, Icarus, $\mathbf{5 6}, 51$.

Zinner, E. 1987, Nature, 330, 730. 